\title{
5. A New Crystalline Form of Carbon under Shock Compression
}

\author{
By Hisako HiraI and Ken-ichi Kondo \\ Research Laboratory of Engineering Materials, \\ Tokyo Institute of Technology, \\ 4259 Nagatsuta, Yokohama 227
}

(Communicated by Syun-iti Aкıмото, м. J. А., March 12, 1991)

\begin{abstract}
A new crystalline carbon form was successfully recovered from the shock-compressed graphite to $65 \mathrm{GPa}$ and $3700 \mathrm{~K}$ using a rapid cooling technique. Electron diffraction pattern (EDP) of the form can not be identified as any kinds of carbon form in the literature. The EDP is characterized by the presence of forbidden reflections indexed as $\{200\}$ and $\{240\}$ in extinction rule of cubic diamond. Other reflections have similar $\mathrm{d}$-spacings to those of cubic diamond, indicating that basic structure of the form is close to cubic diamond.
\end{abstract}

Key words: Diamond-like structure; carbon; shock compression.

Phase relation of elemental carbon has been extensively studied for the past several decades over the wide range of pressure and temperature. Recently, major interest of investigators has tended into carbon behaviors in some restricted pressure-temperature regions. The first is the region under low pressure and high temperature where various phases have been reported by CVD and PVD method. ${ }^{\text {e.g.1),2) }}$ The second is under high pressure and high temperature where the location of the diamond-liquid boundary line is argued.e.g.3) The third is under relatively low temperature and high pressure where a few transitional phases between graphite and hexagonal diamond have been suggested ${ }^{4-6}$ ) after the conversion experiment on hexagonal diamond. ${ }^{7)}$ Besides, in the region around the extraporated line of the graphite-liquid boundary, carbon behavior including a possible amorphization has also attracted attention. The purpose of this study is to develop a rapid cooling technique under shock compression, and to clarify carbon behavior under wide range of P-T conditions. A new crystalline form of solid carbon was found in the sample recovered from the shock-compressed graphite. The results of characterization for the new crystalline carbon form are reported.

A commercial grade graphite (PERMA FOIL) supplied by Toyo Tanso Co., Ltd. was used as starting materials. This is a thin and flexible sheet of graphite, which was produced by chemical, heat, and rolling treatments of natural graphite powder. Apparent density is $1.0 \mathrm{~g} / \mathrm{cm}^{3}$. The thin sheet (less than $100 \mu \mathrm{m}$ in thickness) of the material was placed between $\mathrm{Cu}$ disks. The sample assembly was impacted by $\mathrm{Cu}$-flyer using a two-stage light-gas gun with bore of $20 \mathrm{~mm}$ in diameter. ${ }^{8)}$ The impact velocity was $2.5 \mathrm{~km} / \mathrm{sec}$, comparing to peak pressure of $65 \mathrm{GPa}$. Shock temperature was estimated to be $3700 \mathrm{~K}$ using the previously reported equations ${ }^{8)}$ on the basis of Birch-Murnaghan equation of state. The recovered sample was characterized mainly by a transmission electron microscope (TEM). For TEM observation, specimen was collected at distance less than 20 $\mu \mathrm{m}$ from the interface of $\mathrm{Cu}$ disk and was prepared by crushing.

Typical examples of electron micrographs of the recovered specimen are shown in Figs. 1, 2 and 3. Prior to the detailed TEM observation, the specimen fragments were 
confirmed not to be extra contaminations such as quartz or other silicate formerly pointed out by Smith, ${ }^{9)}$ using an energy dispersive x-ray spectrometer which can detect the presence of light elements heavier than boron. Granular crystals about $1000 \AA$ in size were found in the present experiments (Figs. 1,2 and 3). Large crystals more than $1 \mu \mathrm{m}$ in size were rarely found. These crystals were usually divided into polygonal-shaped domains. Some of them were hexagonal-shaped and were intimately contacted with each other like mosaic tile. The apparent features of the crystal grains were fairly clear; dislocations and/or other defects could not be seen, nor any contrast due to strain could be observed. Partly, the crystals showing sharp fringe contrast likely caused by twin or stacking faults could also be seen.

Fig. 3 is a dark field image generated by using $\{200\}$ reflection described below in detail. Electron diffraction pattern (EDP) from the granular crystals is composed of rings with sharp spots (Fig. 4). A list of the observed d-spacings is given in Table I. The $\mathrm{d}$-spacings of the diffraction rings could not be identified with carbyne, graphite (including compressed graphite), hexagonal diamond and cubic diamond. As for graphite and hexagonal diamond, even considering the special case that each basal plane of the two structure is oriented normal to the electron beam, the EDP observed could not be explained by both forms. The observed d-spacing is close to that of cubic diamond but there is a distinct difference; forbidden reflection rings indexed as $\{200\}$ and $\{240\}$ in the extinction rule for the space group of cubic diamond (Fd3m) are observed with strong intensities. If the (200) reflection appears alone, the possibility that the reflection may be due to double or multiple reflections is not completely ruled out. However, the (240) reflection ring is accompanied with (200) ring, and the (240) ring is located near to the limiting sphere generating diffraction. This means that the presence of the (240) reflection is extremely difficult to be interpreted by double reflection. In addition, when the crystal is tilted around [100] zone axis until other reflections almost disappear, the (200) reflections still remain in keeping strong intensity. It is, therefore, evident that the forbidden reflections of (200) and (240) are not attributed to double reflection but are intrinsic for the crystals.

The appearance of these forbidden reflections indicates a lack of d-glide plane from Fd3m symmetry of cubic diamond. It is proved that the new form has lower symmetry than Fd3m but basic structure may not be so different from that of cubic one. The above-mentioned material is exactly crystalline form consisting of carbon. Its EDP can never belong to any kinds of known carbon form. From the characteristics of the basic structure, morphology and feature, this new crystalline form is called "N-diamond" here.

A numerical analysis based on the Fourier's heat-conduction equation gives cooling rates of $10^{10}$ and $10^{6} \mathrm{~K} / \mathrm{sec}$ at distance of $1 \mu \mathrm{m}$ and $15 \mu \mathrm{m}$ from the interface with the $\mathrm{Cu}$ disk, respectively. The observed specimen was collected from almost interface part with the $\mathrm{Cu}$ disk. Thus, quite rapid cooling might be accomplished in the specimen observed, at latest, $10^{6} \mathrm{~K} / \mathrm{sec}$. The successful quenching of a new crystalline form in the present study may depend mainly on the rapid cooling rate which can not be realized by using conventional static apparatuses.

Recent works on the P-T phase diagram of elemental carbon suggested the occurrence of a certain phase transition or the formation of a few new forms prior to the conversion from graphite to hexagonal diamond. ${ }^{4)-6)}$ Further information on these new crystalline form of carbon was obtained from the in-situ measurements of x-ray diffraction, Raman spectroscopy and optical property of graphite under very high pressure at room temperature using diamond anvil cell. ${ }^{10)-13)}$ Among them, a few experimental observations are agreeable with that of the N-diamond; x-ray diffraction lines observed at $16.4 \mathrm{GPa}$ by Zhao and Spain $^{5)}$ and that at $22.5 \mathrm{GPa}$ by Sugiyama et al. ${ }^{10)}$ are comparable with the 


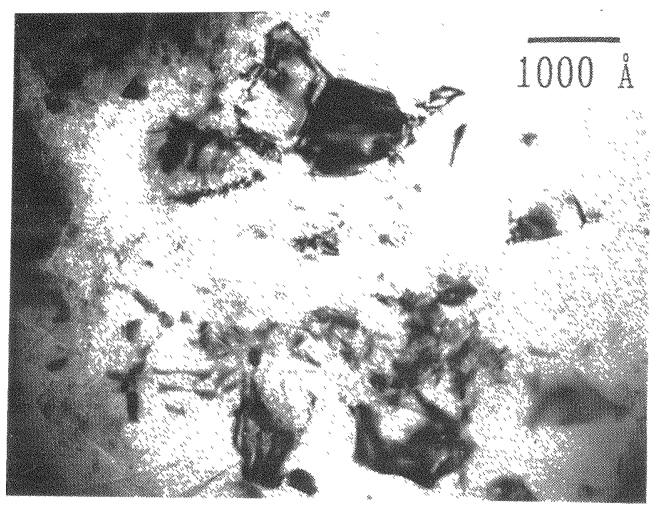

Fig. 1. Electron micrograph of a new crystalline form of carbon. Note that granular crystals are intimately contacted with each other.

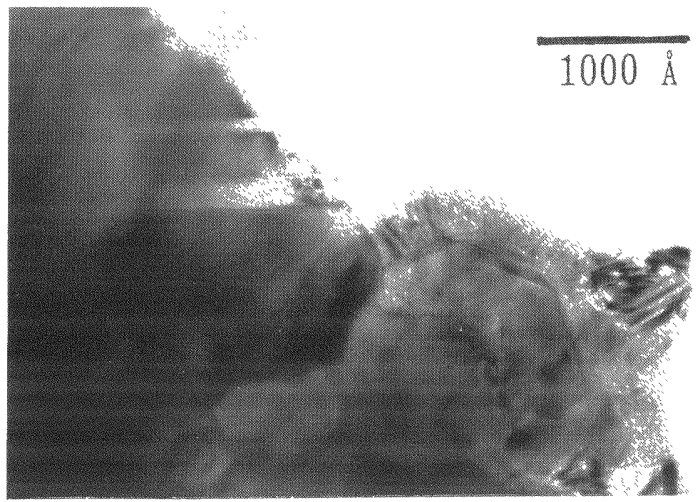

Fig. 2. Bright field image of a new crystalline form of carbon. Polygonal-shaped crystals are found, one of them showing fringe contrast.

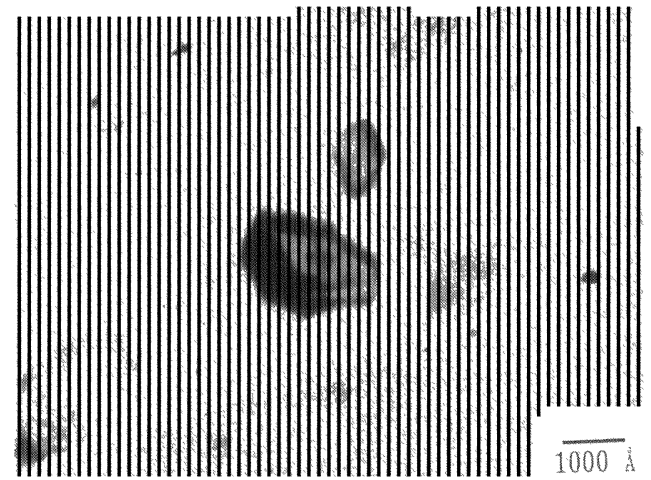

Fig. 3. Dark field image of a new carbon form generated by using forbidden reflection. 


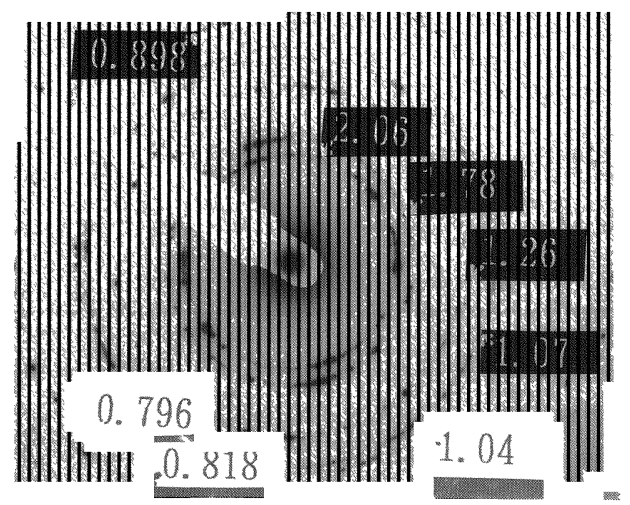

Fig. 4. Electron diffraction pattern of the new crystalline form of carbon.

Table I. Comparison of the observed d-spacing with those of cubic and hexagonal diamond (Lonsdaleite)

\begin{tabular}{cccccccc}
\hline \multicolumn{2}{c}{ Present study } & \multicolumn{3}{c}{ Diamond } & \multicolumn{3}{c}{ Lonsdaleite } \\
$\mathrm{d}-\mathrm{obs}(\AA)$ & Intensity & $\mathrm{d}(\AA)$ & $\mathrm{hkl}$ & Intensity & $\mathrm{d}(\AA)$ & $\mathrm{hkl}$ & Intensity \\
\hline & & & & & 2.19 & 100 & 100 \\
2.06 & $\mathrm{~s}$ & 2.06 & 111 & 100 & 2.06 & 002 & 100 \\
& & & & & 1.920 & 101 & 50 \\
1.78 & $\mathrm{~s}$ & $(1.783$ & $200)^{* *}$ & & & & \\
& & & & & 1.500 & 102 & 25 \\
1.26 & $\mathrm{~s}$ & 1.261 & 220 & 25 & 1.260 & 110 & 75 \\
& & & & & 1.170 & 103 & 50 \\
1.07 & $\mathrm{~s}$ & 1.0754 & 311 & 16 & 1.075 & 103 & 50 \\
1.04 & $\mathrm{~m}$ & 1.0297 & $222^{*}$ & & & & \\
& & & & & 1.055 & 201 & 25 \\
0.898 & $\mathrm{w}$ & 0.8916 & 400 & 8 & & & \\
& & & & & 0.855 & 203 & 25 \\
0.818 & $\mathrm{w}$ & 0.8182 & 331 & 16 & 0.820 & 210 & 25 \\
0.796 & $\mathrm{~m}$ & $(0.7976$ & $240)^{* *}$ & & & & \\
0.726 & $\mathrm{~m}$ & 0.7281 & 422 & & & & \\
0.683 & $\mathrm{~m}$ & 0.6864 & 115 & & & & \\
\hline
\end{tabular}

** forbidden reflection in the space group of $\mathrm{Fd} 3 \mathrm{~m}$

* allowed reflection but intensity is $\sim 0$

characteristic lines of the $\mathrm{N}$-diamond (d:2.06 and $1.78 \AA$ ), in spite that the experimental conditions are quite different. Considering the present shock condition, the $\mathrm{N}$-diamond can be interpreted as a possible intermediate and metastable phase on a way to cubic diamond. The presence of the $\mathrm{N}$-diamond suggests a unique conversion behavior of carbon from graphite to cubic diamond under shock loading.

The authors would like to thank Prof. S. Akimoto, M. J. A., for his valuable comments and communication of this paper to the Japan Academy. 


\section{References}

1) A. G. Whittaker: Science, 200, 763 (1978).

2) H. Vora, and T. J. Moravec: J. Appl. Phys., 52, 6151 (1981).

3) F. P. Bundy: Physica, A 156, 169 (1989).

4) M. Hanfland, H. Beister, and K. Syassen: Phys. Rev., B 39, 12598 (1989).

5) Y. X. Zhao, and I. L. Spain: ibid., B 40, 993 (1989).

6) A. F. Goncharov, I. N. Makarenko, and S. M. Stishov: Sov. Phys. JETP, 69, 380 (1989).

7) F. P. Bundy, and J. S. Kasper: J. Chem. Phys., 46, 3437 (1967).

8) K. Kondo, A. Sawaoka, and T. J. Ahrens: J. Appl. Phys., 52, 5084 (1981).

9) P. P. K. Smith, and P. R. Buseck: Science, 216, 984 (1982).

10) M. Sugiyama et al.: Abst. 31st High Pressure Conf. Jpn., 1A 22, p. 70 (1990).

11) M. Ueno et al.: ibid., 1A 20, p. 66 (1990).

12) W. Utsumi, and T. Yagi: ibid., 1A 19, p. 64 (1990).

13) H. Harashima et al.: ibid., 1A 21, p. 68 (1990). 